\title{
ALTITUDES OF A SIMPLEX IN n-SPACE
}

\author{
SAHIB RAM MANDAN
}

(received 26 October 1959, revised 1 May 1962)

In continuation of the two previous papers $(10 ; 11)$, this paper was originally written at the Indian Institute of Technology, Kharagpur and revised at the University of Sydney under the advice of Prof. T. G. Room. Although the altitudes of a general simplex $\mathrm{S}(A)$ in $n$-space $(n>2)$ do not concur as they do for a triangle $(n=2)$, yet we observe that its Monge point, $M(1 ; 5)$, is an appropriate analogue of the orthocentre of a triangle such that $M$ coincides with its orthocentre when it is orthogonal (or orthocentric). In consistency with the previous papers $(10 ; 11 ; 13 ; 15)$ we shall call $M$ as the S-point of $\mathrm{S}(A)$ and denote it as $S$ as explained in $\S 1.2$. The altitudes of $\mathrm{S}(A)$ are all met by the $(n-2)$-spaces normal to its plane faces at their orthocentres, each parallel to $\left(\begin{array}{c}n \\ 3\end{array}\right)$ of them, thus indicating the associated character of the altitudes as discussed separately in 2 other papers $(12 ; 16)$. Before we introduce an orthogonal simplex and develop its properties in regard to its $\gamma$-altitudes and associated hyperspheres, we come across a number of intermediate ones of special interest. Two special types are treated here and the other two are developed in 2 other papers $(13 ; 15)$.

\section{General simplex}

1.1. General behaviour of altitudes. Let $\mathrm{S}(A)=A_{0} \cdots A_{n}$ be a general simplex; in this paper the same letter will be used to represent a point and its position vector relative to its circumcentre, 0 , and $x$ will be used as the position vector of a current point. The primes normal to the sides of a triangle $A_{i} A_{j} A_{k}$ from its. respective opposite vertices are then given by the equations

$$
\begin{aligned}
\left(A_{j}-A_{k}\right) \cdot\left(x-A_{i}\right)=0, \quad\left(A_{k}-A_{i}\right) \cdot\left(x-A_{\jmath}\right)=0, & \\
& \left(A_{i}-A_{\jmath}\right) \cdot\left(x-A_{k}\right)=0 .
\end{aligned}
$$

These primes are obviously coaxal and their common $(n-2)$-space, $s_{i j k}$, is normal to the plane face $A_{i} A_{j} A_{k}$ at its orthocentre. Also $s_{i j k}$ meets the 3 altitudes of $\mathrm{S}(A)$ from $A_{i}, A_{j}, A_{k}$ and is parallel to the remaining $n-2$ altitudes. Thus follows 
THEOREM 1. The altitudes of a general $n$-simplex are met by the $(n-2)$ spaces normal to its plane faces at their orthocentres, each parallel to $\left(\begin{array}{l}n \\ 3\end{array}\right)$ of them.

For each altitude is normal to the $\left(\begin{array}{c}n \\ 3\end{array}\right)$ plane faces of $\mathrm{S}(A)$ formed by its $n$ vertices lying in its corresponding prime face.

1.2. S-point. a. Coxeter [5] has proved that the Monge planes of an $n$ simplex $\mathrm{S}(A)$, defined as the primes normal to its edges from the centroid of its opposite $(n-2)$-faces, concur at its Monge point, $M$ (orthocentre for $n=2$ ) which lies on its Euler line joining its circumcentre, $O$, to its centroid, $G$, such that $M G: G O=2:(n-1)$. For $n=4$, we arrived at the same point in a different way (10), called it as the S-point of the simplex and denoted it as $S$. In consistency with the same, we shall call $M$ as the S-point of $\mathrm{S}(A)$ and denote it as $S$. We then have by definition the relation

$$
(n+1) G=(n-1) S
$$

and deduce the analogous

TheORem 2. The normals to the prime faces of an $n$-simplex $(n>2)$ at the points, which divide the joins of their S-points to the feet of its respective altitudes in the ratio $1:(n-2), C O N C U R$ at its S-point.

Proof. Let $G_{i}, S_{i}$ be the centroid and the S-point of the prime face, $\mathrm{S}_{i}$, of $\mathrm{S}(A)$ opposite its vertex $A_{i} ; G_{i n}, A_{i n}, T_{i}, O_{i}$ be the projections * of $G, A_{i}, S, O$ in $\mathrm{S}_{i} . O_{i}$ is then the circumcentre of $\mathrm{S}_{i}$ and $A_{i n}$ is the foot of the altitude of $\mathrm{S}(A)$ from $A_{i}$ to $\mathrm{S}_{i}$ such that

$$
(n+1)\left(G-G_{i n}\right)=\left(A_{i}-A_{i h}\right)
$$

and

$$
(n+1) G_{i h}=(n-1) T_{i}+2 O_{i} .
$$

Again by definition we have

$$
n G_{i}=2 O_{i}+(n-2) S_{i}=(n+1) G-A_{i}
$$

Now from these 3 relations we derive

$$
(n-1) T_{i}=(n-2) S_{i}+A_{i n} .
$$

That is, $T_{i}$ is the point on the join $S_{i} A_{i n}$ dividing it in the ratio $1:(n-2)$ such that the normal to the prime $S_{i}$ at $T_{i}$ passes through $S$. Hence follows the proposition which could be proved independently by induction.

b. Let $A_{s h}$ be the foot of the altitude of the simplex $\mathrm{S}(A)$ from its vertex $A_{j}$ to its opposite prime face $\mathrm{S}_{j} ; A_{i h j}, A_{j h i}$ be the projections of $A_{i h}, A_{j h}$ in

- Here as well as henceforward projections are Orthogonal unless otherwise stated. 
its $(n-2)$-face $\mathrm{S}_{i j}$ common to $\mathrm{S}_{i}, \mathrm{~S}_{j}$ such that $A_{i n j}$ is the foot of the altitude of $\mathrm{S}_{j}$ from $A_{i}$ and $A_{j h i}$ of $\mathrm{S}_{i}$ from $A_{j} ; S_{j}$ be the S-point of $\mathrm{S}_{j}$ and $T_{j}$ the projection of $S$ in $S_{j} ; S_{i j}$ be the S-point of $\mathrm{S}_{i j}$ and $T_{i s}, T_{j s}$ be the points dividing the joins of $S_{i j}$ to $A_{j h i}, A_{i n j}$ in the ratio $1:(n-3)$ as the projections of $S_{i}, S_{j}$ in $S_{i j}$. The common point $T_{i j s}$ of the joins $T_{i s} A_{i h j}, T_{j s} A_{j h i}$ divides them in the ratio $1:(n-2)$ as the common projection, in $S_{i j}$, of the points $T_{i}, T_{j}$ dividing the joins $S_{i} A_{i n}, S_{j} A_{j h}$ in the same ratio (Fig. $1, i=n, j=n-1$ ).

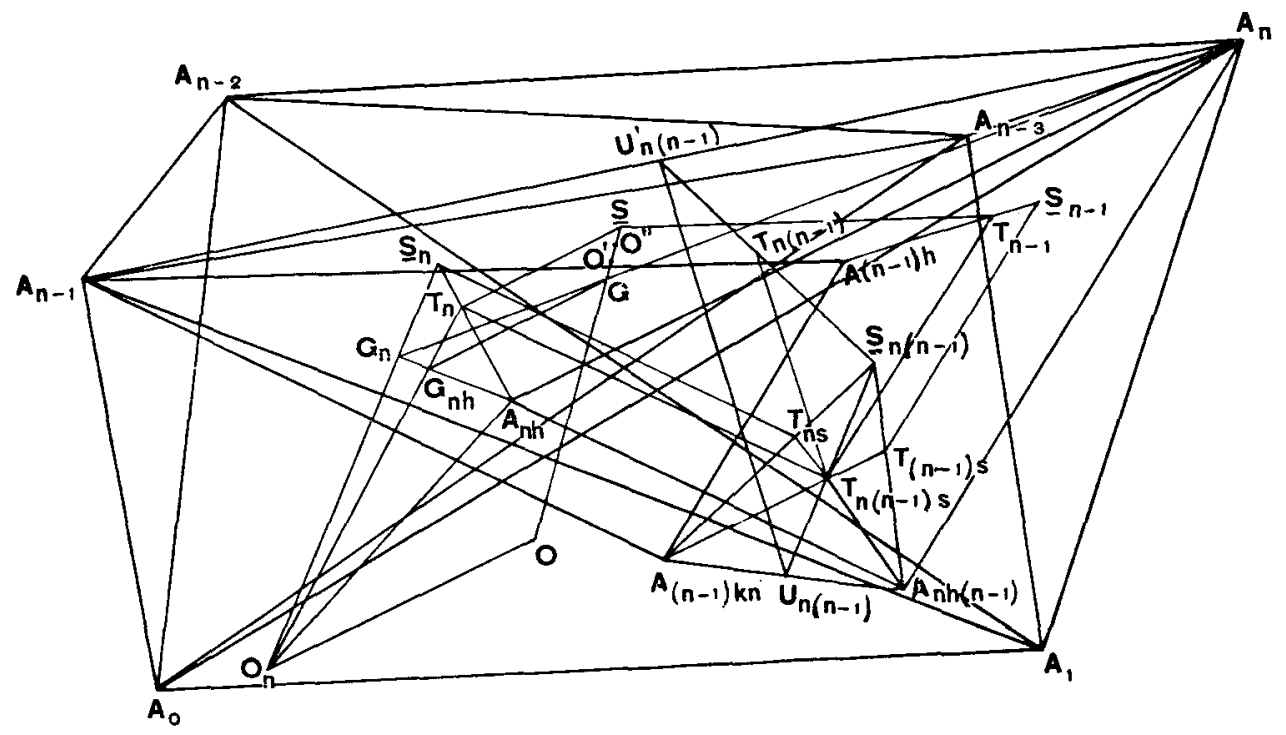

Fig. 1.

For

(vi)

$$
(n-3) S_{i j}=(n-2) T_{i s}-A_{j h i}=(n-2) T_{j s}-A_{i n j}
$$

and therefore

$$
(n-1) T_{i j s}=(n-2) T_{i s}+A_{i h j}=(n-2) T_{j s}+A_{j h i}
$$

The plane $T_{i} T_{j} T_{i j s}$ is then normal to $S_{i j}$ at $T_{i j s}$ and therefore contains the normals to $\mathrm{S}_{i}, \mathrm{~S}_{j}$ at $T_{i}, T_{j}$ meeting in $S$. Again from the relations (vi), (vii) we have

$$
(n-3) S_{i j}=(n-1) T_{i j s}-2 U_{i j} \quad\left(\text { where } 2 U_{i j}=A_{i n s}+A_{j h i}\right)
$$

Thus $S_{i j} T_{i j s}$ meets the join $A_{i n j} A_{j h i}$ in its midpoint $U_{i j}$. The plane, through $U_{i j}$, parallel to the 2 parallel planes $A_{i} A_{i h} A_{i h j}, A_{j} A_{j h} A_{j h i}$ then meets the edge $A_{i} A_{j}$ of $\mathrm{S}(A)$ in its midpoint $U_{i j}^{\prime}$, and the plane $T_{i} T_{j} T_{i j s}$ meets the join $S_{i j} U_{i j}^{\prime}$ in the point $T_{i j}$ dividing the same in the ratio $2:(n-3)$ in which it divides $S_{i j} U_{i j}$ at $T_{i j s}$ (Fig. $1, i=n, j=n-1$ ). That is, the plane normal to the $(n-2)$-face $\mathrm{S}_{i j}$ of $\mathrm{S}(A)$ from $T_{i j}$ contains its $\mathrm{S}$-point $S$. Thus we have 
THEOREM 3. The planes normal to the $(n-2)$-faces of an $n$-simplex from points, dividing the joins of their S-points to the midpoints of its opposite edges in the ratio $2:(n-3)$, CONCUR at its S-point.

1.3. Associated hyperspheres. a. If the circum-radius of the $\operatorname{simplex} \mathrm{S}(A)$ be taken as a unit, the vectorial equation of its circumhypersphere $(O)$ is

$$
(O) \equiv x^{2}-1=0 .
$$

The centroid $G_{i}$ of the face $\mathrm{S}_{i}$ is related to $A_{i}$ by (iv) as

$$
n\left(G-G_{i}\right)=\left(A_{i}-G\right)
$$

so that the equation of the hypersphere $\left(O^{\prime}\right)$ containing the centroids $G_{i}$ of the faces $S_{i}$ is obtained from that of $(O)$ by the homothetic transformation

$$
x=(n+1) G-n x^{\prime}
$$

i.e., the equation is

$$
\left(O^{\prime}\right) \equiv\{n x-(n+1) G\}^{2}-1=0 .
$$

The centre $O^{\prime}$ is given by

$$
n O^{\prime}=(n+1) G=(n-1) S
$$

from (i). I.e. the external and internal centres of similitude $(9)$ of $(O)$ and $\left(O^{\prime}\right)$ are $S$ and $G$ respectively, Hence we have

TheOREM 4. The $n+1$ points $P_{i}$, dividing the joins of the S-point of an $n$-simplex to its vertices in the ratio $1:(n-1)$, and their $n+1$ projections in its corresponding prime faces lie on its hypersphere $\left(O^{\prime}\right)$, called therefore as its ' $3(n+1)$ point-sphere' (cf. $1 ; 4 ; 10)$.

For every join $P_{i} G_{i}$ is a diameter of $\left(O^{\prime}\right)$ as evident from the following relations:

$$
n\left(P_{i}+G_{i}\right)=(n-1) S+A_{i}+n G_{i}=n O^{\prime}+(n+1) G=2 n O^{\prime}
$$

b. The equations of the 2 hyperspheres $(S),(G)$ of antisimilitude and one of similitude, $\left(O^{\prime \prime}\right)$, of $(O),\left(O^{\prime}\right)$ with centres at $S, G, O^{\prime \prime}=(S+G) / 2$ are by given
(xiv)
$(S) \equiv n x^{2}-2 n S \cdot x+(n-1) S^{2}+1=0$,$$
(G) \equiv n x^{2}-2 n G \cdot x+(n+1) G^{2}-1=0 \text {, }
$$

$$
\left(O^{\prime \prime}\right) \equiv x^{2}-(G+S) \cdot x+G \cdot S=0 .
$$

$(S)$ is referred to as the quasi-polar hypersphere $(2 ; 10),(G)$ as the $G$-sphere, and $\left(O^{\prime \prime}\right)$ as the GS-sphere (with $G S$ as diameter) of $\mathrm{S}(A)$. Thus we have 
THEOREM 5. With every $n$-simplex are associated 5 coaxal hyperspheres $(O),\left(O^{\prime}\right),\left(O^{\prime \prime}\right),(G),(S)$ like 5 such spheres associated with a tetrahedron or 5 such circles associated with a triangle, viz., its circumcircle and q-point circle, their 2 circles of antisimilitude (one being its polar circle, the other with centre at is centroid is called its g-circle) and their circle of similitude, called its $l$-circle, with centres on its Euler line (8).

c. As an immediate consequence we now have the following:

Theorem 6. The porer of the S-point of an n-simplex w.r.t. its circumhypersphere is equal to $n$ times the square of the radius of its quasi-polar hypersphere (cf. $2 ; 10$ ).

THEOREM 7. Every hypersphere with a median of a simplex (join of a vertex to the centroid of its opposite prime face) as diameter is orthogonal to its quasi-polar hypersphere.

That is, the radius $\left(S^{2}-1\right) / n$ of $(S)$ is equal to the power of $S$ w.r.t. the former hypersphere

$$
\left(A_{i} G_{i}\right) \equiv x^{2}-\left(A_{i}+G_{i}\right) \cdot x+A_{i} \cdot G_{i}=0 .
$$

It now follows from the relations (i), (iv), (ix). For $A_{i}$ lies on $(O)$ and therefore $A_{i}^{2}=1$.

THEOREM 8. The GS-sphere of a simplex is the 'radical hypersphere' of its quasi-polar hypersphere and G-sphere (7).

It follows from the relations (xiv) to (xvi) by reducing the equations of $(S)$ and $(G)$ into their standard form by making the coefficient of $x^{2}$ unity. For then $(S)+(G)=\left(O^{\prime \prime}\right)$.

THEOREM 9. The polar of a vertex $A_{i}$ of an n-simplex w.r.t. its quasi-polar hypersphere cuts the hypersphere $\left(A_{i} G_{i}\right)$ with its corresponding median as diameter in an $(n-2)$-sphere on its $3(n+1)$ point-sphere and that of its the centroid $G_{i}$ of its corresponding prime face on its circumhypersphere (cf. 10).

It follows from the relations (i), (iv) and the equations (ix), (x), (xiv), (xvii).

THEOREM 10. Every hypersphere, with centre at a vertex $A_{i}$ or the centroid $G_{i}$ of the opposite prime face of an n-simplex, orthogonal to its quasi-polar hypersphere is coaxal with its $G$-sphere and the hypersphere $\left(A_{i} G_{i}\right)$ of the Theorem 9 (cf. 10).

It can be proved by the use of equations (xiv), (xv), (xvii). But geometrically it is almost obvious when we observe that the 3 hyperspheres under consideration are all orthogonal to $(S)$ and have their centres collinear in the median $A_{i} G_{i}$. Hence their common radical prime is one normal to $A_{i} G_{i}$ through $S$ and is thus uniquely determined. 
THEOREM 11. The simplex $\mathrm{S}(B)$ formed of the diametrically opposite points $B_{i}$ of the vertices $A_{i}$ of a simplex $\mathrm{S}(A)$ is homothetic to that formed of the centroids $G_{i}$ of its prime faces w.r.t. its S-point with homothetic ratio equal to $n$.

For from the relations (i), (iv) we have

$$
(n-1) S=(n+1) G=n G_{i}+A_{i}=n G_{i}-\left(-A_{i}\right)=n G_{i}-B_{i} .
$$

REMARK. $A_{i}, G_{i}$ form a pair of conjugate points for the quasipolar hypersphere $(S)$ by the Theorem 7 . Now the Theorem 9 follows geometrically almost obviously from the Theorems 4 and 11 . For a diameter subtends a right angle at every point of its sphere and the polar of a point $P$ w.r.t. a hypersphere is at right angles to the join of $P$ to its centre passing thorough all its conjugate points (see 10).

\section{Biorthocentric simplex}

2.1. Existance. When the altitudes of a simplex $\mathrm{S}(A)$ (§ 1.1) from its 2 vertices $A_{i}, A_{j}$ meet in a point $H^{\prime}$, it is said to be Biorthocentric (BoS) with $H^{\prime}$ as its Biorthocentre. If $O_{i}, O_{j}$ be'the vectors representing the normals from the circumcentre $O$ of the $\operatorname{BoS}$ to its prime faces $\mathrm{S}_{i}, \mathrm{~S}_{j}$, for some $P_{i}, P_{j}$ we then have

$$
H^{\prime}=A_{i}+P_{i} O_{i}=A_{j}+P_{j} O_{j}
$$

and also

$$
O_{i} \cdot A_{k}=c_{i}(k \neq i), O_{j} \cdot A_{k}=c_{j}(k \neq j)
$$

where $c_{i}, c_{j}$ are some constants. It follows that

$$
\left(P_{i} O_{i}-P_{j} O_{j}\right) \cdot A_{k}=P_{i} c_{i}-P_{j} c_{j}
$$

and therefore that

$$
\left(A_{i}-A_{j}\right) \cdot\left(A_{k}-A_{l}\right)=0(k, l \neq i, j),
$$

i.e., the edge $A_{i} A_{j}$ is normal to the opposite $(n-2)$-face $\mathrm{S}_{i j}$ of the BoS. Conversely if (xxi) holds, we can deduce the existance of $H^{\prime}$.

Further we may observe geometrically almost obviously (see 10) that if the common normal secant of $A_{i} A_{j}$ and $\mathrm{S}_{i j}$ meet $A_{i} A_{j}$ in $U^{\prime}$ and $\mathrm{S}_{i j}$ in $U, U U^{\prime}$ is an altitude of the triangle $A_{i} A_{j} U$, the other two being along $A_{i} H^{\prime}, A_{j} H^{\prime}$ meeting $A_{j} U, A_{i} U$ respectively in $A_{i h}, A_{j h}$ such that $A_{i} A_{i h}, A_{j} A_{j h}$ are the altitudes of the $\operatorname{BoS}$ to $\mathrm{S}_{i}, \mathrm{~S}_{j}$ and $A_{i} U, A_{j} U$ are the altitudes of $\mathrm{S}_{j}, \mathrm{~S}_{i}$ to $\mathrm{S}_{i j}$ (Fig. 2, $i=n, j=n-1$ ).

$U U^{\prime}$ is called the special bialtitude of the BoS to its special edge $A_{i} A_{j}$ and its special $(n-2)$-face. We then have 


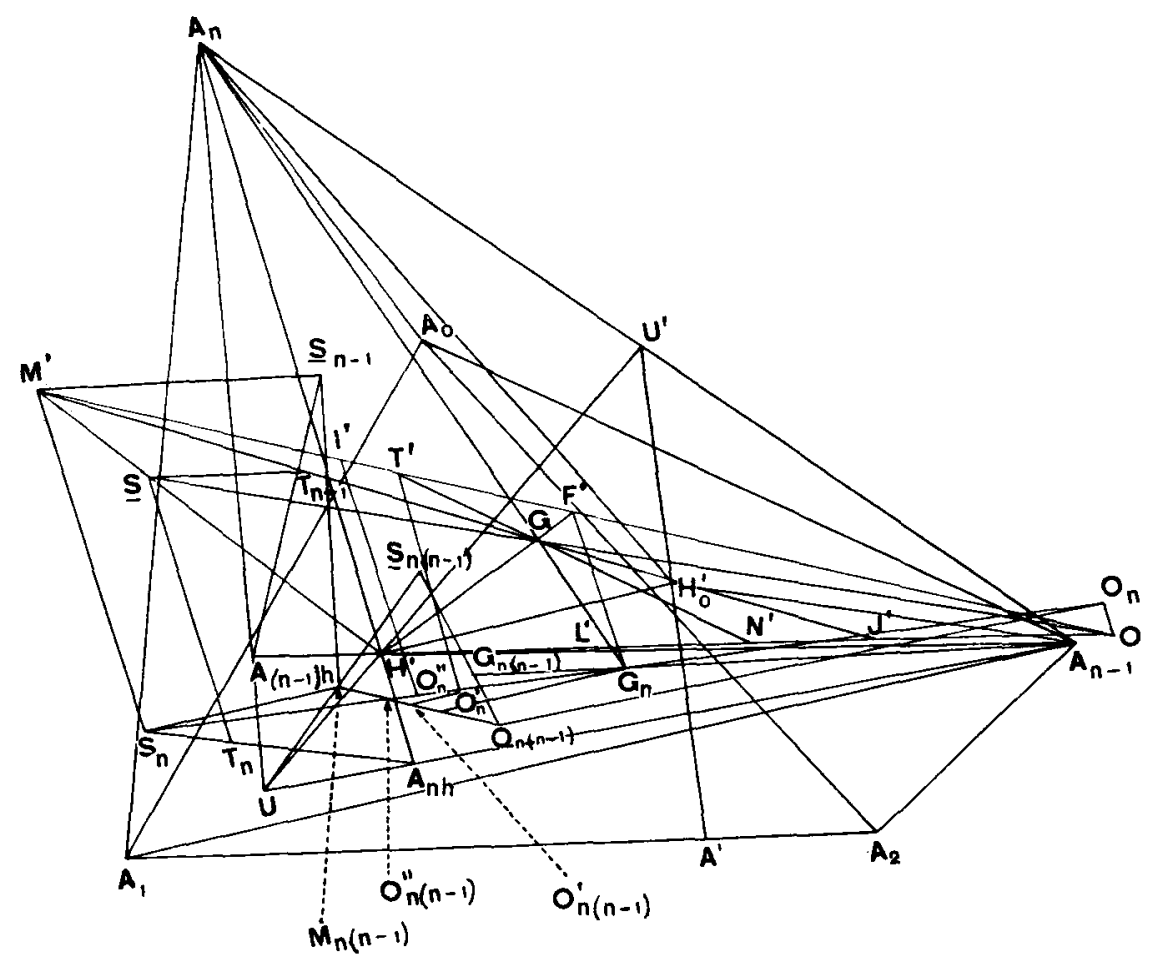

Fig. 2.

TheOREM 12. An $n$-simplex $\mathrm{S}(A)$ becomes $B o S$, if and only if, an edge $A_{i} A_{j}$ of $\mathrm{S}(A)$ be normal to its opposite $(n-2)$-face $\mathrm{S}_{i j}$, and then its 2 altitudes from its vertices $A_{i}, A_{j}$ CONCUR with its special bialtitude $U U^{\prime}$ at its Biothocentre, such that their feet lie respectively on the altitudes of its prime faces opposite $A_{i}, A_{j}$ to $\mathrm{S}_{i j}$ and the feet of the later altitudes coincide at the foot $U$ of $U U^{\prime}$ in $\mathrm{S}_{i j}$.

2.2. Orthocentres of faces through special edge of BoS. $A_{i} A_{j}$ being the special edge of the $\mathrm{BoS}$, its $n-1$ prime faces $\mathrm{S}_{k}$ through $A_{i} A_{j}$ are then all obviously Biorthocentric such that their altitudes from $A_{i}, A_{j}$ meet at their respective Biorthocentres $H_{k}^{\prime}$. If $H^{\prime}$ be the Biorthocentre of the BoS, we have the following relations:

$$
\begin{array}{cc}
\left(A_{i}-H^{\prime}\right) \cdot\left(A_{j}-A_{\imath}\right)=0 & (l \neq i), \\
\left(A_{j}-H^{\prime}\right) \cdot\left(A_{i}-A_{\imath}\right)=0 & (l \neq j), \\
\left(A_{i}-H_{k}^{\prime}\right) \cdot\left(A_{j}-A_{l}\right)=0 & (l \neq i, k), \\
\left(A_{j}-H_{k}^{\prime}\right) \cdot\left(A_{i}-A_{l}\right)=0 & (l \neq j, k) .
\end{array}
$$

From these relations follow two more as follows: 
(xxii)

$$
\begin{gathered}
\left(H^{\prime}-A_{l}\right) \cdot\left(A_{i}-A_{j}\right)=0 \quad(l \neq i, j)_{s} \\
\left(H^{\prime}-H_{k}^{\prime}\right) \cdot\left(A_{p}-A_{l}\right)=0(p=i, j ; l \neq k) .
\end{gathered}
$$

That is, the prime normal to $A_{i} A_{j}$ from $H^{\prime}$ passes through its special $(n-2)$-face $\mathrm{S}_{i j}$ such that the projection of its every vertex $A_{l}$ in $\mathrm{S}_{i j}$ on $A_{i} A_{j}$ coincides with that of $H^{\prime}$, which projects into $H_{k}^{\prime}$ in $\mathrm{S}_{k}$ (Fig. 2, $k=0$ ). Thus follows

THEOREM 13. Every $r$-face $(r>3)$ of a BoS in an n-space through its special edge $A_{i} A_{j}$ is Biorthocentric such that the Biorthocentre $H^{\prime}$ of the Bos projects into that of its every such face; its every tetrahedron $A_{i} A_{j} A_{k} A_{l}$ is semi-orthocentric (3) such that $H^{\prime}$ projects into its semi-orthocentre relative to $A_{i} A_{j}$ and into the orthocentre of its every triangle $A_{i} A_{j} A_{k}$; the altitudes of all its triangles $A_{i} A_{1} A_{k}$ and the special bialtitudes of all its other r-faces through $A_{i} A_{j}$ to $A_{i} A_{j}$ CONCUR with its special biallitude at their common foot on $A_{i} A_{j}$ (cf. 10).

2.3. S-, $M$ - and F-point of BoS. From Fig. 2, Theorem 2 and relations (iv) and (v) it follows that the point $M^{\prime}$, called the $M$-point of the BoS, given by the relation

(xxiv)

$$
(n-2) M^{\prime}=(n-1) S-H^{\prime},
$$

( $S$ being the S-point and $H^{\prime}$ the Biorthocentre of the BoS), projects into the S-points $S_{i}, S_{j}$ of its prime faces $S_{i}, S_{j}$, and its $F$-point $F^{\prime}$, defined by the relation

$$
n F^{\prime}=(n-2) M^{\prime},
$$

projects into their centroids. From (i), (xxiv) and (xxv) also follows that (xxvi)

$$
n F^{\prime}=(n+1) G-H^{\prime},
$$

( $G$ being the centroid of the BoS).

Now the existance of $M^{\prime}$ implies that $k_{i}$ and $k_{f}$ exist such that

$$
M^{\prime}=S_{i}+k_{i} O_{i}=S_{j}+k_{j} O_{j}(\S 2.1) \text {. }
$$

Or, $(n+1) G-A_{i}-2 O_{i}+(n-2) k_{i} O_{i}=(n+1) G-A_{j}-2 O_{j}+(n-2) k_{j} O_{s}$ by (iv). I.e., by (xviii) we have $P_{i}=2-(n-2) k_{i}, P_{j}=2-(n-2) k_{i}$. These values of $k_{i}, k_{j}$ give us (xxiv) by (iv) and (xviii).

Similarly $F^{\prime}$ exists if we can find $\sigma_{i}, \sigma_{j}$ from the relations

$$
F^{\prime}=G_{i}+\sigma_{i} O_{i}=G_{j}+\sigma_{j} O_{j}\left(G_{i} G_{j} \text { being the centroids of } \mathrm{S}_{i}, \mathrm{~S}_{j}\right) .
$$

Or, $(n+1) G-A_{i}+n \sigma_{i} O_{i}=(n+1) G-A_{j}+n \sigma_{j} O_{j}$ by (iv). I.e., by (xviii) we have $n \sigma_{i}=-\rho_{i}, n \sigma_{j}=-\rho_{j}$.

These values of $\sigma_{i}, \sigma_{j}$ and relations (iv), (xviii) give us (xxvi). 
Conversely the existance of $M$ - or $F$-point of a simplex can be similarly proved to imply that of its Biorthocentre. Thus we have

ThEOREM 14. The following conditions to make an n-simplex BoS are equivalent: (a) An edge $A_{i} A_{j}$ is normal to its opposite $(n-2)$-tace $\mathrm{S}_{i j}$, (Th. 13). (b) Any of the following pairs of normals to a pair of its prime faces $\mathrm{S}_{i}, \mathrm{~S}_{\text {; }}$ concur

(i) through $A_{i}, A$, in its Biorthocentre $H^{\prime}$,

(ii) at their centroids $G_{i}, G_{j}$ in its $F$-point $F^{\prime}$,or,

(iii) at their S-points $S_{i}, S_{j}$ in its $M$-point $M^{\prime}$.

2.4. Special $(n-2)$-sphere. The special edge $A_{i} A_{i}$ of the BoS being normal to its opposite $(n-2)$-face $S_{i j}$, the mediater $m$ of its special bialtitude $U U^{\prime}$ (i.e., the prime normal to $U U^{\prime}$ at its mid-point) cuts its non-special edges $A_{i} A_{k}, A_{j} A_{k}$ at their midpoints lying on an $(n-2)$-sphere, called its special $(n-2)$-sphere. For obviously they form a right $(n-1)$-prism whose two $(n-2)$-faces are parallel to $\mathrm{S}_{i j}$ and their $n-1$ connecting edges to $A_{i} A_{j}$ all equal to $\frac{1}{2} A_{i} A_{j}$. In fact, Prof. Room has shown that this $(n-2)$ sphere lies on the hypersphere $\left(x-\frac{1}{2} H^{\prime}\right)^{2}=$ constant. For it passes through the $2(n-1)$ points $\frac{1}{2}\left(A_{i}+A_{k}\right), \frac{1}{2}\left(A_{j}+A_{k}\right)$ as follows: The point $\frac{1}{2}\left(A_{i}+A_{k}\right)$ $=\frac{1}{2}\left(H^{\prime}-\rho_{i} O_{i}+A_{k}\right)$ lies on it if

$$
\begin{aligned}
\left(\rho_{i} O_{i}-A_{k}\right)^{2} & =\rho_{i}^{2} O_{i}^{2}-2 \rho_{i} c_{i}+1=\rho_{i}^{2} O_{i}^{2}-2 \rho_{i} O_{i} \cdot A_{j}+1 \text { (by relation (xix)) } \\
& =\left(\rho_{i} O_{i}-A_{j}\right)^{2}=\left(\rho_{j} O_{j}-A_{i}\right)^{2}=\left(H^{\prime}-A_{i}-A_{j}\right)^{2}=\text { constant }
\end{aligned}
$$

which is true. Hence we have

TheOREM 15. The mid-points of the $2(n-1)$ non-special edges $A_{i} A_{k}$, $A_{j} A_{k}$ through 2 vertices of an $n$-simplex lie on its special $(n-2)$-sphere, if and only if it becomes BoS with $A_{i} A_{j}$ as its special edge $(3 ; 10)$.

2.5. $N-, J-, L$ - and $H$-spheres. a. From the properties and relationship of the 5 coaxal circles associated with a triangle and their centres on its Euler line $(\S 1.3 \mathrm{~b})$ we may deduce from the Theorem 13 the following

THEOREM 16. The q-point, $g$-, $l$-, and polar circles of the $n-1$ plane faces through an edge $A_{i} A_{j}$ of an $n$-simplex lie respectively on an hypersphere, called its $N-, J$-, $L$ - and $H$-sphere $\left(N^{\prime}\right),\left(J^{\prime}\right),\left(L^{\prime}\right)$ and $\left(H^{\prime}\right)$, belonging to a coaxal family $f($ say) to which belongs its circumhypersphere $(O)$ too, if and only if it becomes a $B o S$ with $A_{i} A_{j}$ as its special edge such that the radical axes of the $n-1$ coaxal families of circles associated with them CONCUR on $A_{i} A_{3}$ determining the radical prime of $f ;\left(N^{\prime}\right)$ passes through its special $(n-2)$-sphere.

b. From the property of the polar circle of a triangle we have the following apparent

TheOREM 17. The polars of the vertices $A_{i}, A$, of the BoS of Theorem 16 
w.r.t. its $H$-sphere $\left(H^{\prime}\right)$ are its respective opposite primes, and therefore the feet $U, U^{\prime}$ of its special bialtitude form a pair of inverse points for $\left(H^{\prime}\right)$.

For the centre of $\left(H^{\prime}\right)$ obviously lies at its Biorthocentre $H^{\prime}$, and $U, U^{\prime}$ are therefore (Th. 12) collinear in a diameter of $\left(H^{\prime}\right)$ normal to $A_{i} A_{j}$ and its opposite $(n-2)$-face polar to $A_{i} A_{j}$ for $\left(H^{\prime}\right)$.

c. The centres $O, H^{\prime}, N^{\prime}, J^{\prime}, L^{\prime}$ of the 5 hyperspheres $(O),\left(H^{\prime}\right),\left(N^{\prime}\right),\left(J^{\prime}\right)$, $\left(L^{\prime}\right)$ are vectorially related as follows ( $O$ being the origin):

$$
2 N^{\prime}=H^{\prime} ; 3 L^{\prime}=2 H^{\prime} ; 3 J^{\prime}=H^{\prime} .
$$

From the the third relation of (xxvii) and (xxv), (xxvi) we have (xxviii)

$$
(n-2) M^{\prime}=(n+1) G-3 J^{\prime}
$$

leading to the following

Theorem 18. The centre of the J-sphere of a BoS is collinear with its centroid and its $M$-point, as well as with its circumcentre, Biorthocentre and the centres of its $N$ - and L-spheres.

2.6. $M-, F_{-}, I$ - and $T$-spheres. The S-points $S_{i}, S_{j}$ of the prime faces $\mathrm{S}_{i}, \mathrm{~S}_{j}$ of the BoS (§2.3) project into the same point $M_{i j}$, in their common $(n-2)$-face $S_{i j}$, where it meets the plane $S_{i} M^{\prime} S_{j}$ (Fig. $2, i=n, j=n-1$ ), on the join of its S-point $S_{i j}$ to the foot of the special bialtitude of the BoS therein, and the diameters of their $(3 n)$ point spheres $\left(O_{i}^{\prime}\right),\left(O_{j}^{\prime}\right)$ (Th. 5) through its centroid $G_{i j}$ into the same diameter of the $(n-3)$-sphere section of either by it. That is, $\left(O_{i}^{\prime}\right),\left(O_{j}^{\prime}\right)$ lie on an hypersphere $\left(T^{\prime}\right)$ with centre $T^{\prime}$ on $O M^{\prime}$ such that

(xxix)

$$
(n-1) T^{\prime}=(n-2) M^{\prime}
$$

For if $O_{i}^{\prime}$ be the centre of $\left(O_{i}^{\prime}\right)$ as the projection of $T^{\prime}$ in $S_{i}$, the relation (xiii) becomes

$$
(\mathrm{n}-1) O_{i}^{\prime}=(n-2) S_{i}+O_{i}\left(O_{i} \text { being the circumcentre of } S_{i}\right) .
$$

Consequently the 5 coaxal $(n-2)$-spheres associated with $S_{i}$ or $S_{j}$ have the same 5 coaxal $(n-3)$-sphere sections by $\mathrm{S}_{i j}$ with the join of $M_{i j}$ to its circumcentre $O_{i j}$ as their line of centres. Again from (xxvii), (xxviii), (xxix) we have

$(\mathbf{x x x})$

$$
(n-1) T^{\prime}=(n+1) G-2 N^{\prime} .
$$

Thus follow the following

THEOREM 19. The quasi-polar $(n-2)$-spheres, G-, GS- and (3n)pointspheres $(\S 1.3 \mathrm{~b})$ of 2 prime faces of an $n$-simplex lie respectively on an hypersphere, called its $M-, F-, I$ - and $T$-sphere $\left(M^{\prime}\right),\left(F^{\prime}\right),\left(I^{\prime}\right)$ and $\left(T^{\prime}\right)$, belonging to a coaxal family $f^{\prime}$ (say) to which belongs its circumhypersphere $(O)$ too, if and 
only if it becomes a BoS. The centres of $\left(M^{\prime}\right),\left(F^{\prime}\right)$ lie at its $M$ - and $F$-points $M^{\prime}, F^{\prime}$ and that of $\left(I^{\prime}\right)$ at the midpoint of $M^{\prime} F^{\prime}$.

THEOREM 20. The centroids of the $2 n-1(n-2)$-faces of an n-simplex in its 2 prime faces lie on its $T$-sphere $\left(T^{\prime}\right)$, if and only if it becomes BoS such that $\left(T^{\prime}\right)$ is homothetic to its $N$-sphere $\left(N^{\prime}\right)$ w.r.t. its centroid $G$ with homothetic ratio $-2:(n-1)$ and therefore $G$ lies on the join of their centres $T^{\prime}, N^{\prime}$.

\section{Semi-orthocentric simplex}

3.1. r-Orthocentric simplex. a. If a pair of consecutive edges $A_{i} A_{j}, A_{j} A_{k}$ of an $n$-simplex $\mathrm{S}(A)$ be both special ( $(2.1)$, they are rectangular to all their respictive opposite edges, in particular to $A_{k} A_{l}, A_{i} A_{l}$ respectively. Thus $A_{i} A_{j} A_{k} A_{l}$ form an orthocentric or orthogonal tetrahedron and $A_{k} A_{i}$ is normal to the opposite $(n-2)$-face $\mathrm{S}_{k i}$ of $\mathrm{S}(A)$ such that $A_{i} A_{j} A_{k} A_{l} A_{m}$ form a semi-orthocentric 4-simplex (11). Consequently follows from Theorem 12 the following

THEOREM 21. If a pair of consecutive edges $A_{i} A_{j}, A_{j} A_{k}$ of an $n$-simplex $\mathrm{S}(A)$ be both special, so is also $A_{k} A_{i}$, their plane $s_{2}$ is then normal to its opposite $(n-3)$-space $s_{n-3}$, its $n-2$ tetrahedra $A_{i} A_{j} A_{k} A_{l}$ are all orthocentric and $\left({ }_{2}^{n-2}\right)$ 4-simplexes $A_{i} A_{j} A_{k} A_{l} A_{m}$ are all semi-orthocentric. Or, if 2 altitudes of $\mathrm{S}(A)$ meet a third, the three CONCUR, say at $H_{3} . \mathrm{S}(A)$ is then said to be 3 -orthocentric with $H_{3}$ as its 3 -orthocentre, $s_{2}$ and $s_{n-3}$ as its special elements, and denoted as $\mathrm{S}_{3}(A)$.

b. As an immediate consequence now follow the following

THEOREM 22. (i) If $r-1(r<n-1)$ consecutive edges of an $(n+1)$ gen $s$ formed of the vertices of a simplex $\mathrm{S}(A)$ in an $n$-space be special, all its edges and planes in their $(r-1)$-space $s_{r-1}$ are special, $s_{r-1}$ is then normal to its opposite $(n-r)$-space $s_{n-r}$ and its $r$ altitudes from its vertices in $s_{r-1}$ CONCUR, say at $H_{r} . \mathrm{S}(A)$ is then said to be $r$-orthocentric with $H_{r}$ as its $r$-orthocentre, $s_{r-1}$ and $s_{n-r}$ as its special elements, and denoted as $\mathrm{S}_{r}(A)$.

(ii) If, further, the opposite $n-r$ edges of $s$ be also special, the other $n-r+1$ altitudes of $\mathrm{S}_{r}(A)$ also CONCUR, say at $H_{n-r+1}$. It is then said to be Semi-orthocentric (SoS) with $H_{r}, H_{n-r+1}$ as the pair of its Semi-orthocentres, $s_{r-1}$ and $s_{n-r}$ as its principal elements, and denoted as $s_{r \cdot(n-r+1)}$. That is, if all but a pair of opposite edges of s be special, $\mathrm{S}(A)$ becomes an SoS.

TheOREM 23. Every $\mathrm{S}_{n-1}(A)$ in an $n$-space or $(n-1)$-orthocentric $n$ simplex is Biorthocentric too and therefore forms an SoS $\mathrm{S}_{(n-1) \cdot 2}$. For its other 2 altitudes also MEET.

THEOREM 24. Every $\mathrm{S}_{n}(A)$ in an $n$-space or $n$-orthocentric $n$-simplex is Orthogonal (OS) such that all its altitudes CONCUR at its Orthocentre. 
THEOREM 25. The $(n-r+1)$-simplexes and $(\underset{2}{n-r+1})(r+1)$-simplexes of an $\mathrm{S}_{r}(A)$ having its special $(r-1)$-face common are all respectively Orthogonal and Semi-orthocentric.

THEOREM 26. A subsimplex of an SoS is either Orthogonal or Semiorthocentric, but every prime face of an $\mathrm{OS}$ is ever Orthogonal with Orthocentre at the foot of the altitude of $O S$ to it.

3.2. $p$-Altitudes. a. The unique normal secant of a $(p-1)$-space of an $n$-simplex and its opposite $(n-p)$-space is called its $p$-or $(n-p+1)$ altitude (Bialtitude for $p=2$ ) to them.

The $r$-space joining the special $(r-1)$-space $s_{r-1}$ of an $n$-simplex $S_{r}(A)$ to its $r$-orthocentre $H_{r}$ is obviously normal to its opposite special $(n-r)$ space $s_{n-r}$ meeting the same in $U$ (say) such that the $r$-simplex $\left(s_{r}\right)$ formed of $U$ and the $(r-1)$-simplex of $S_{r}(A)$ in $s_{r-1}$ is orthogonal with $r$ altitudes same as the concurrent ones of $\mathrm{S}_{r}(A)$. Thus $H_{r}$ is the orthocentre of $\left(s_{r}\right)$ and $U H_{r}$ is its $(r+1)$ th altitude meeting $s_{r-1}$ in $U^{\prime}$ (say). $U U^{\prime}$ is then no other than the $r$-altitude of $\mathrm{S}_{r}(A)$ to $s_{r-1}$ and is called special. Again the altitudes of the $(n-r+1)$-simplexes of $\mathrm{S}_{r}(A)$, having its $(n-r)$-simplex in $s_{n-r}$ common, to $s_{n-r}$ concur at $U$. Thus from what precedes follow the following

Theorem 27. The $\left(\begin{array}{c}r \\ p\end{array}\right)$ p-altitudes of an n-simplex $\mathrm{S}_{r}(A)$ to its $(p-1)$ spaces in its special $(r-1)$-space are all special and pass through its $r$ orthocentre, and each meets its opposite $(n-p)$-space $s_{n-p}$ in a point where CONCUR the altitudes of its $p(n-p+1)$-simplexes, having its $(n-p)$ simplex in $s_{n-p}$ common, to $s_{n-p}$.

THEOREM 28. Both the Semi-orthocentres of an SoS $\mathrm{S}_{r \cdot(n-r+1)}$ lie on its special $r$ - or $(n-r+1)$-altitude, referred to as its 'principal' $r$-altitude.

b. We may also prove the following

THEOREM 29. If $U, U^{\prime}$ be the feet of a p-altitude of an $n$-simplex in its relative $(p-1)$-space $s_{p-1}$ and $(n-p)$-space $s_{n-p}$, and $U U^{\prime}$ meet its altitude from a vertex $A_{i}$ in $s_{n-p}$, the p-altitude of its prime face opposite $A_{i}$ to $s_{p-1}$ meets $s_{p-1}$ in $U$ and the opposite $(n-p-1)$-space in $U^{\prime \prime}$ such that $A_{i}$, $U^{\prime}, U^{\prime \prime}$ are collinear in an altitude of its $(n-p)$-face in $s_{n-p}$.

c. Let an $(r-1)$-face $\left(s_{r-1}\right)$ of an $n$-simplex $\mathrm{S}(A)$ be Orthogonal with Orthocentre at $U^{\prime}, U U^{\prime}$ its $r$-altitude to its $(r-1)$-space $s_{r-1}$ of $\left(s_{r-1}\right)$ meeting its opposite $(n-r)$-space $s_{n-r}$ in $U, U^{\prime} U^{\prime \prime}$ the bialtitude of $\left(s_{r-1}\right)$ to its edge $A_{i} A_{j}$ meeting its opposite $(r-3)$-space $s_{r-3}$ in $U^{\prime \prime}, A_{k}$ a vetex of $\mathrm{S}(A)$ in $s_{n-r}$ and $A_{l}$ in $s_{r-3}$ such that $s_{r-1}$ is normal to $s_{n-r}$. We then have the following vectorial relations

$$
\begin{aligned}
\left(A_{i}-A_{j}\right) \cdot\left(U-U^{\prime}\right)=0 & =\left(A_{i}-A_{j}\right) \cdot\left(U^{\prime}-U^{\prime \prime}\right)=\left(A_{i}-A_{j}\right) \cdot\left(U-A_{k}\right) \\
& =\left(A_{i}-A_{j}\right) \cdot\left(U^{\prime \prime}-A_{l}\right)
\end{aligned}
$$


from which we deduce

$$
\left(A_{i}-A_{j}\right) \cdot\left(U-U^{\prime \prime}\right)=0=\left(A_{i}-A_{j}\right) \cdot\left(A_{k}-A_{l}\right) .
$$

Thus $A_{i} A_{j}$ is normal to its opposite $(n-2)$-space determined by $s_{n-r}$, $s_{r-3}$. Hence follows

THEOREM 30. An n-simplex $\mathrm{S}(A)$ becomes $\mathrm{S}_{r}(A)$, if and only if an $(r-1)$ face $\left(s_{r-1}\right)$ of it is Orthogonal with Orthocentre at the foot $U^{\prime}$ of its $r$-altitude to its $(r-1)$-space $s_{r-1}$ of $\left(s_{r-1}\right)$ therein meeting its opposite $(n-r)$-space $s_{n-r}$ in $U$, and $s_{r-1}$ is normal to $s_{n-r}$. If further its $(n-r)$-face in $s_{n-r}$ be also Orthogonal with Orthocentre at $U$, it becomes an SoS with $U U^{\prime}, s_{r-1}, s_{n-r}$ as its principal elements.

3.3. Orthocentres. From the preceding section follow now the following

THEOREM 31. A special p-altitude of an n-simplex $\mathrm{S}_{r}(A)$ meets its relative $(n-p)$-face, which is $(r-p)$-orthocentric, in its $(r-p)$-orthocentre.

THEOREM 32. The prime faces of an n-simplex $\mathrm{S}_{r}(A)$ opposite its vertices in its special $(r-1)$-space $s_{r-1}$ are all $(r-1)$ orthocentric such that the feet of its altitudes therein lie at their respective $(r-1)$-orthocentres; its q-faces having $p$ vertices in $s_{r-1}$ are all $p$-orthocentric (Orthogonal for $p=q-1$ ) such that its $r$-orthocentre projects in them into their respective p-orthocentres (Orthocentres for $p=q-1$ ).

THEOREM 33. The $(n-2)$-spaces normal to the plane faces, through $r-1$ concurrent edges of an $n$-simplex or $r-1$ consecutive edges of an $(n+1)$ gon formed of its vertices, at their orthocentres CONCUR at $H_{r}($ say), if and only if it becomes $\mathrm{S}_{r}(A)$ with $H_{r}$ as its $r$-orthocentre, (cf. Ths. 13, 21, 22).

THEOREM 34. Every Orthocentre of every order of every face of an SoS is an orthogonal projection of its one or the other Semi-otthocentre.

3.4. S-point. Now we prove by induction the following

THEOREM 35. The S-point of an SoS $\mathrm{S}_{r \cdot(n-r+1)}$ lies on its principal $r$ altitude dividing the segment between its $r$-and $(n-r+1)$-orthocentres in the ratio $(n-r):(r-1)$.

Proof. Let $A_{i}$ be a vertex of the SoS in its principal $(r-1)$-space and $A_{j}$ in its principal $(n-r)$-space; $\mathrm{S}_{i}, \mathrm{~S}_{j}$ its prime faces opposite $A_{i}, A_{j}$ and $S_{i}, S_{j}$ their S-points; $H, H^{\prime}$ its $r$ - and $(n-r+1)$-orthocentres, $H_{i}, H_{i}^{\prime}$ their projections in $\mathrm{S}_{i}$ and $H_{j}, H_{j}^{\prime}$ in $\mathrm{S}_{j} . \mathrm{S}_{i}$ is then Semi-orthocentric, $H_{i}$, $H_{i}^{\prime}$ are its $(r-1)$ - and $(n-r+1)$-orthocentres and $H_{i}$ is the foot of the altitude of the SoS to it (Th. 32). $\mathrm{S}_{j}$ too is Semi-orthocentric, $H_{j}, H_{j}^{\prime}$ are its $r$ - and $(n-r)$-orthocentres and $H_{j}^{\prime}$ is the foot of the altitude of the SoS to it. If $r=2, S_{i}$ is Orthogonal with Orthocentre at $H_{i}^{\prime}$ coincident with $S_{i}$. 
If $r=n-1, S_{j}$ is Orthogonal with Orthocentre at $H_{j}$ coincident with $S_{j}$.

Assuming the proposition to be true for an $(n-1)$-simplex, $S_{i}, S_{j}$ lie on $H_{i} H_{i}^{\prime}, H_{j} H_{j}^{\prime}$ such that

$(\mathrm{xxxi})$

$$
\begin{aligned}
& (n-2) S_{i}=(r-2) H_{i}+(n-r) H_{i}^{\prime}, \\
& (n-2) S_{j}=(r-1) H_{j}+(n-r-1) H_{j}^{\prime} .
\end{aligned}
$$

If $T_{i}, T_{j}$ be on $H_{i} H_{i}^{\prime}, H_{j} H_{j}^{\prime}$ such that

(xxxii)

$$
(n-1) T_{x}=(r-1) H_{x}+(n-r) H_{x}^{\prime}(x=i, j),
$$

the normals to $S_{x}$ at $T_{x}$ concur on the principal $r$-altitude (Fig. 3) of the SoS at its S-point $S$ by definition (Th. 2) such that

(xxxiii)

$$
(n-1) S=(r-1) H+(n-r) H^{\prime}
$$

For $T_{x}$. satisfy the relations

$$
(n-1) T_{i}=(n-2) S_{i}+H_{i},(n-2) T_{j}=(n-2) S_{j}+H_{j}^{\prime}
$$

by virtue of (xxxi), (xxxii).

Thus if the proposition is true for an $(n-1)$-simplex, it is true for an

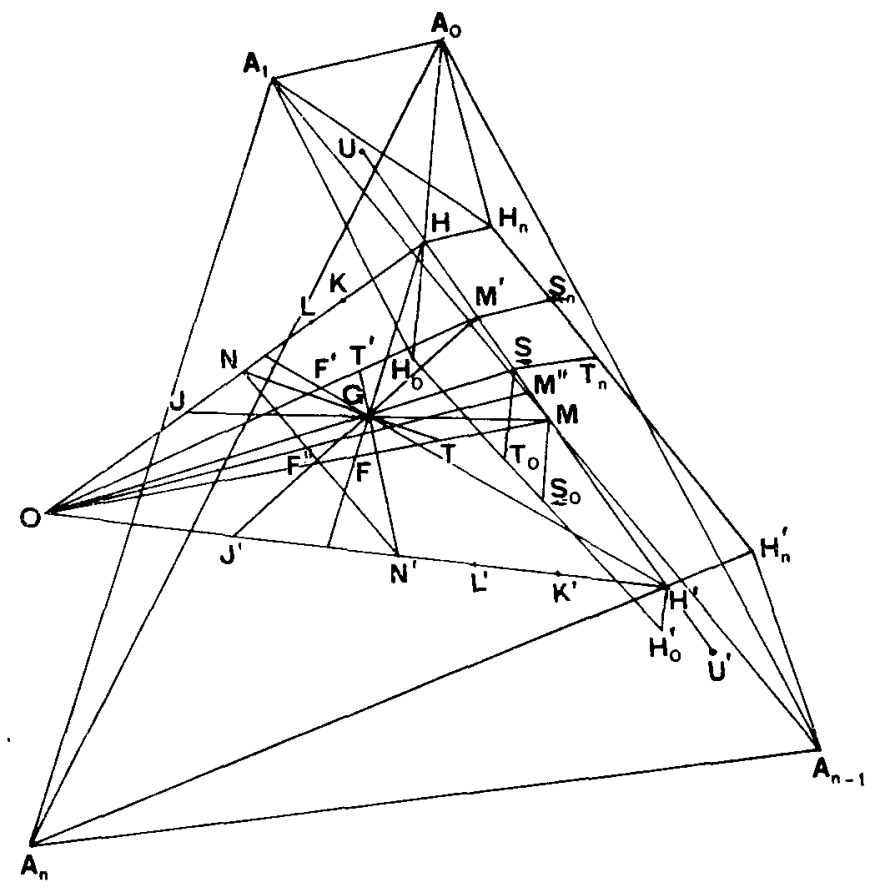

Fig. 3. 
$n$-simplex. But we know it to be true for a 4 -simplex (11) and hence it holds for an $n$-simplex for successive values of $n=5,6,7 \cdots$.

3.5. $M$-points. From the preceding proposition follows

THeorem 36. (i) The normals to the $r$ prime faces of an SoS $\mathrm{S}_{r \cdot(n-r+1)}$, opposite its vertices in its principal $(r-1)$-space $s_{r-1}$, at their S-points all CONCUR with its principal $r$-altitude $\propto$ at its $M$-point $M$ which then divides the segment between its $r$-and $(n-r+1)$-orthocentres $H, H^{\prime}$ in the ratio $(n-r):(r-2)$ such that $M$ coincides with $H^{\prime}$ if $r=2$, and those to its other primes CONCUR at its other $M$-point $M^{\prime}$ dividing $H H^{\prime}$ in the ratio $(n-r-1):(r-1)$ such that $M^{\prime}$ coincides with $H$ if $r=n-1$.

(ii) The $\left(\begin{array}{c}v \\ p\end{array}\right)\left(\begin{array}{c}n-r+1 \\ q\end{array}\right) \quad(p+q)$-spaces normal to its Semi-orthocentric $(n-p-q)$ faces, with $r-p$ vertices in $s_{r-1}$, at their $\mathrm{S}$-points all CONCUR with $\alpha$ at its $M$-point $M_{q}^{p}\left(M_{0}^{1}=M, M_{1}^{0}=M^{\prime}\right)$, dividing $H H^{\prime}$ in the ratio $(n-q-r):(r-p-1)$, where then CONCUR the $\left(\begin{array}{c}r \\ u\end{array}\right)\left(\begin{array}{c}n-r+1 \\ t\end{array}\right)(u+t)$ spaces normal to its $(n-u-t)$-faces, with $r-u(u \leqq p, t \leqq q)$ vertices in $s_{r-1}$, at their $M$-points $M_{q-t}^{p-u}$.

(iii) In particular, the $\left(\begin{array}{c}r \\ 2\end{array}\right)\left(\begin{array}{c}n-r+1 \\ 2\end{array}\right)(n-3)$-spaces normal to its Semiorthocentric tetrahedral faces at their Monge points all CONCUR at the midpoint $M^{\prime \prime}=M_{n-r-1}^{r-2}$ of $H H^{\prime}$.

(iv) The distances of its $M$-points $M_{q}^{p}$ from $H$ or $H^{\prime}$ for fixed $q$ or $p$ form an harmonic progression to which belongs the distance of its S-point too for $q$ or $p=0$.

3.6. F-points. a. Again as an immediate consequence of what precedes $(\S \S 3.5,1.2)$ follows.

THEOREM 37. The $\left(\begin{array}{c}r \\ p\end{array}\right)\left(\begin{array}{c}n-r+1 \\ q\end{array}\right)(p+q)$-spaces normal to the $(n-p-q)$ faces of an SoS $S_{r \cdot(n-r+1)}$, with $r-p$ vertices in its principal $(r-1)$-face $s_{r-1}$, at their centroids all CONCUR on the join of its circumcentre $O$ and its $M$-point $M_{q}^{p}\left(M_{q}^{r}=M_{q}^{r-1}=H^{\prime}, M_{n-r+1}^{p}=M_{n-r}^{p}=H\right)$ dividing the same in the ratio $(n-p-q-1): 2$ at its $F$-point $F_{q}^{p}\left(F_{0}^{1}=F, F_{1}^{0}=F^{\prime}\right)$ where CONCUR the $\left(\begin{array}{c}r \\ u\end{array}\right)\left(\begin{array}{c}n-r+1 \\ t\end{array}\right)(u+t)$-spaces normal to its $(n-u-t)$-faces, with $r-u$ vertices in $s_{r-1}(u \leqq p, t \leqq q)$, at their $F$-points $F_{q-t}^{p-u}$ (cf. $\S 2.3$ ).

In particular, the $\left(\begin{array}{c}r \\ 2\end{array}\right)\left(\begin{array}{c}n-r+1 \\ 2\end{array}\right)(n-3)$-spaces normal to its Semi-orthocentric tetrahedral faces at their centroids all CONCUR at the midpoint $F^{\prime \prime}=F_{n-r-1}^{r-2}$ of $O M^{\prime \prime}$ (Fig. 3).

b. Further it can be readily seen that all the $F$-points $F_{0}^{p}(p \neq r)$ of the SoS are collinear with its centroid $G$ and its $r$-orthocentre $H$, and $F_{q}^{0}(q \neq$ $n-r+1)$ with $G$ and its $(n-r+1)$-orthocentre $H^{\prime}$. Thus follows the following

TheOREM 38. The S-point and $M$-points $M_{0}^{p}$ of the SoS project from its circumcentre $O$ on the join of its centroid $G$ to its $r$-orthocentre $H$ into $G$ and its 
$F$-points $F_{0}$ such that their distances from $H$ form an harmonic progression, and $M_{q}^{0}$ project from $O$ on the join of $G$ to its $(n-r+1)$-orthocentre $H^{\prime}$ into $F_{q}^{0}$ such that their distances from $H^{\prime}$ similarly form an harmonic progression to which belongs the distance of $G$ too from $H^{\prime}$ (cf. Th. 36 (iv)).

3.7. Principal $(n-2)$-sphere. We may now prove the following (cf. § 2.4)

THEOREM 39. The midpoints of the $r(n-r+1)$ non-special edges of an SoS $\mathrm{S}_{r \cdot(n-r+1)}$ form a SIMPLOTOPE (6) of the type $(r-1, n-r)$ in the mediator of its principal r-altitude having $\left(\begin{array}{c}r \\ 2\end{array}\right)\left(\begin{array}{c}n-r+1 \\ 2\end{array}\right)$ plane faces as rectangles, one in each of its semi-orthocentric (3) tetrahedral face, and therefore lie on an $(n-2)$-sphere, referred to as PRINCIPAL, with centre at its F-point $F^{\prime \prime}$ (Th. 37).

3.8. $N$ - and $J$-spheres. From Theorems 16, 33 follow the following

THEOREM 40. The q-point and g-circles of the $\left(\begin{array}{c}r \\ 3\end{array}\right)+\left(\begin{array}{c}r \\ 2\end{array}\right)(n-r+1)$ triangular faces $(r>2)$ of an n-simplex $\mathrm{S}_{r}(A)$ through its special edges lie respectively on its $N$ - and $J$-sphere $(N),(J)$ with centres $N, J$ at the midpoint and the point of trisection of the join of its circumcentre $O$ to its $r$-orthocentre $H$. $(N)$ passes through the first 12-point sphere (8) of its Orthogonal tetrahedra and the first 20-point 3-sphere (11) of its Orthogonal A-simplexes $(r>3)$.

THEOREM 41. With every SoS. $\dot{S}_{r \cdot(n-r+1)}$ are associated a pair of $N$-spheres $(N),\left(N^{\prime}\right)$ and a pair of $J$-spheres $(J),\left(J^{\prime}\right)$ such that their centres $N, N^{\prime}, J, J^{\prime}$ lie at its $F$-points $N=F_{n-r}^{r-3}=F_{n-r+1}^{r-4}, N^{\prime}=F_{n-r-2}^{r-1}=F_{n-r-3}^{r}, J=F_{n-r+1}^{r-3}$ $=F_{n-r}^{r-2}, J^{\prime}=F_{n-r-1}^{r-1}=F_{n-r-2}^{r}$ for sensible values of $n$ and $r .(N),\left(N^{\prime}\right)$ have its principal $(n-2)$-sphere common with their radical hypersphere. $N N^{\prime}$ is parallel to its principal r-altitude and $J J^{\prime}$ such that the joins of $J, J^{\prime}$ to its corresponding $M$-points $M, M^{\prime}$ meet in its centroid dividing them in the ratio $3:(n-2)$ (Ths. 37,18$)$.

3.9. Semi-polar hyperspheres. From Theorems 16, 17 follows

THEOREM 42. The $\left(\begin{array}{l}r \\ 3\end{array}\right)+\left(\begin{array}{c}r \\ 2\end{array}\right)(n-r+1)$ polar circles $(r>2)$ of the plane faces of an SoS $\mathrm{S}_{r \cdot(n-r+1)}$ through its edges in its principal $(r-1)$-face $\left(s_{r-1}\right)$ and the $n-r+1$ polar $(r-1)$-spheres of its Orthogonal $r$-faces through $\left(s_{r-1}\right)$ all lie on an $H$-sphere $(H)$ with centre at its $r$-orthocentre $H$; the polar circles of its other triangles and the $r$ polar $(n-r)$-spheres of its Orthogonal $(n-r+1)$-faces through its principal $(n-r)$-face all lie on another $\left(H^{\prime}\right)$ with centre at its $(n-r+1)$-orthocentre $H^{\prime}$; the radical prime of $(H),\left(H^{\prime}\right)$ coincides with the mediator of its principal r-altitude whose feet form the pair of their limiting points, and they are referred to as its semi-polar hyperspheres.

3.10. $M$-spheres. From Theorems 19, 36 follows

THEOREM 43. The $\left(\begin{array}{c}r \\ p\end{array}\right)\left(\begin{array}{c}n-r+1 \\ q\end{array}\right)$ quasipolar $(n-p-q-1)$-spheres of the 
Semi-orthocentric $(n-p-q)$-faces of an $\operatorname{SoS~S}_{r \cdot(n-r+1)}$, with $r-p$ vertices in its principal $(r-1)$-face, all lie on an $M$-sphere $\left(M_{q}^{p}\right)$ with centre at its $M$-point $M_{q}^{p}$.

3.11. $I$ - and F-spheres. a. From Theorems 19, 37 follows

THEOREM 44. The $\left(\begin{array}{c}r \\ p\end{array}\right)\left(\begin{array}{c}n-r+1 \\ q\end{array}\right) G$-spheres of the $(n-p-q)$-faces of an SoS $\mathrm{S}_{r \cdot(n-r+1)}$, with $r-p$ vertices in its principal $(r-1)$-space $s_{r-1}$, all lie on an $F$-sphere $\left(F_{q}^{p}\right)$ with centre at its $F$-point $F_{q}^{p}$, and their $G S$-spheres on an $I$-sphere $\left(I_{q}^{p}\right)$ with centre $I_{q}^{p}$ at the midpoint of the join of its corresponding $F$ and $M$-points $F_{q}^{p}, M_{q}^{p}$.

b. We have seen above the 4 particular examples of the $F$-spheres in Theorem 41. Those of the $I$-spheres we have in the following (Th. 16)

THEOREM 45. (i) The $\left(\begin{array}{c}r \\ 3\end{array}\right)+\left(\begin{array}{c}r \\ 2\end{array}\right)(n-r+1)$ l-circles $(r>2)$ of the plane faces of the SoS through its edges in $s_{r-1}$ all lie on an $L$-sphere $(L)=$ $\left(I_{n-r+1}^{r-3}\right)=\left(I_{n-r}^{r-2}\right)$, with centre $L$ at the midpoint of the join of its $r$-orthocentre $H$ to the centre $J$ of its corresponding $J$-sphere $(J)$, which therefore passes through the second 12-point spheres $(1 ; 8)$ of its Orthogonal tetrahedra having three or all the four vertices in $s_{r-1}$; the l-circles of its other triangles and the second 12-point spheres of its other Orthogonal tetrahedra all lie on another $L$-sphere $\left(L^{\prime}\right)=$ $\left(I_{n-r-1}^{r-1}\right)=\left(I_{n-r-2}^{r}\right)$ with centre $L^{\prime}$ at the midpoint of the join of $i t s(n-r+1)$ orthocentre $H^{\prime}$ to the centre $J^{\prime}$ of its second $J$-sphere $\left(J^{\prime}\right)$.

(ii) The $\left(\begin{array}{l}r \\ 5\end{array}\right)+\left(\begin{array}{l}r \\ 4\end{array}\right)(n-r+1)$ 15-point 3-spheres $(r>4)$ of the Orthogonal 4-simplexes of the SoS having four or all the five vertices in $s_{r-1}$ all lie on an hypersphere, referred to as its $k-S P H E R E,(k)=\left(I_{n-r}^{r-3}\right)=\left(I_{n-r+1}^{r-4}\right)$ with centre $k$ at the midpoint of the join of $H$ to the centre $N$ of its corresponding $N$-sphere $(N)$, and those of its other Orthogonal 4-simplexes lie on another $k$-sphere $\left(k^{\prime}\right)=\left(I_{n-r-2}^{r-1}\right)=\left(I_{n-r-3}^{r}\right)$ with centre $k^{\prime}$ at the midpoint of the join of $H^{\prime}$ to the centre $N^{\prime}$ of its second $N$-sphere $\left(N^{\prime}\right)$ (11).

3.12. T-spheres. From Theorems 19, 20, 41, 45 follow

THEOREM 46. (i) The $\left(\begin{array}{c}r \\ p\end{array}\right)\left(\begin{array}{c}n-r+1 \\ q\end{array}\right) 3(n-p-q+1)$ point-spheres of the $(n-p-q)$-faces of an SoS $\mathrm{S}_{r \cdot(n-r+1)}$, with $r-p$ vertices in its principal $(r-1)$-space $s_{r-1}$, all lie on a $T$-sphere $\left(T_{q}^{p}\right)$ with centre $T_{q}^{p}$ on the join of $i t s$ circumcentre $O$ to its $M$-point $M_{q}^{p}$ dividing the same in the ratio $(n-p-q-1): 1$ such that $\left(T_{n-r}^{r-2}\right),\left(T_{n-r-1}^{r-1}\right)$ coincide with its $N$-spheres $(N),\left(N^{\prime}\right) ;\left(T_{n-r}^{r-3}\right)$, $\left(T_{n-r-2}^{r-1}\right)$ with its L-spheres $(L),\left(L^{\prime}\right) ;\left(T_{n-r}^{r-4}\right),\left(T_{n-r-3}^{r-1}\right)$ with its $k$-spheres $(k),\left(k^{\prime}\right)$.

(ii) The $\left(\begin{array}{c}r \\ p\end{array}\right)\left(\begin{array}{c}n-r+1 \\ q+1\end{array}\right)+\left(\begin{array}{c}r \\ p+1\end{array}\right)\left(\begin{array}{c}n-r+1 \\ q\end{array}\right)$ centroids of the $(n-p-q-1)$-faces of the SoS, with $r-p$ or $r-p-1$ vertices in $s_{r-1}$, all lie on $\left(T_{q}^{p}\right)$ which is then homothetic to its $T$-sphere $\left(T_{n-q-r}^{r-p-1}\right)$ w.r.t. its centroid $G$ with homothetic ratio $-(p+q+1):(n-p-q)$ such that $G$ lies on the join of their centres dividing the same in the same ratio 
3.13. Coaxal families. The semi-polar hyperspheres of an SoS may be considered as its limiting $M$-spheres $(H)=\left(M_{n-r}^{p}\right)=\left(M_{n-r+1}^{p}\right), \quad\left(M_{q}^{r}\right)=$ $\left(M_{q}^{r-1}\right)=\left(H^{\prime}\right)(\S \S 3.4,3.5)$. From Theorems 16, 19, 41-46 now follows the following

Theorem 47. The corresponding $M-, F$-, $I$ - and $F$-spheres $\left(M_{q}^{p}\right),\left(F_{q}^{p}\right)$, $\left(I_{q}^{p}\right),\left(T_{q}^{p}\right)$ of an SoS and its circumhypersphere $(0)$ form a coaxal family, and there are 2 such special families such that to one determined by $(0)$ and $a$ semi-polar hypersphere belong an $N-, J$-, $L$ - and $k$-sphere.

\section{Orthogonal simplex $O(S)$}

4.1. Properties. We have already noticed how an OS appears (Ths. 24-26) along with a number of its specialties as its distinguishing features $(\S \S 3.2-3.4)$. But we may consider an $O S$ as a limiting SoS when the pair of its Semi-orthocentres and consequently its S-point as well as its $M$-points coincide at the orthocentre of the $O S$. Thus we enumerate its properties in following

THEOREM 48. Every face of an OS is Orthogonal and every edge special; all its $p$-altitudes CONCUR at its Orthocentre and meet its relative $(p-1)$ - and $(n-p)$-faces in their respective Orthocentres; the altitudes of its $n-1$ plane faces through its every edge $e$ and the bialtitudes of all its other faces through $e$ to $e$ all CONCUR at their common foot on $e$.

THEOREM 49. (i) The centroid $G$ of an $O S$ in an $n$-space is collinear with its Orthocentre $H$ and its circumcentre $O$ dividing $O H$ in the ratio $(n-1): 2$ (§ 1.2a).

(ii) The $\left(\begin{array}{c}n+1 \\ p\end{array}\right)$ p-spaces normal to its $(n-p)$-spaces at their centroids all CONCUR at its F-point $F_{p}$ on its Euler line OGH dividing $O H$ in the ratio $(n-p-1): 2$, and therefore the distances of $F_{p}(p=1, \cdots, n-1$; $\left.F_{n-1}=O\right)$ from $H$ form an harmonic progression $\left(\S 3.6, F_{q}^{p}=F_{p}^{q}=F_{p+a}\right.$ ) such that the $\left(\begin{array}{c}n+1 \\ 3\end{array}\right)(n-2)$-spaces normal to its plane faces at their q-point centres CONCUR at the midpoint $N=F_{n-3}$ of $O H$ where CONCUR the $\left(\begin{array}{c}n+1 \\ 4\end{array}\right)(n-3)$-spaces normal to its solid faces at their centroids.

Or the relationships are expressed vectorially as follows:

(xxxiv)

$$
\begin{aligned}
(n+1) G & =(n-1) H \\
(n-p+1) F_{p} & =(n-p-1) H .
\end{aligned}
$$

4.2. Polar hypersphere. We may now have

THEOREM 50. (i) An SoS in an n-space becomes an OS, if and only if all its $M$-spheres and the pair of its semi-polar hyperspheres coincide with its quasi- 
polar hypersphers into the CONJUGATE (1) or POLAR hypersphere $(H)$ of the $O S$ with centre at its orthocentre $H$ such that $O S$ is self-polar for $(H)$ and the power of $H$ w.r.t. its circumhypersphere $(O)$ is $n$ times the square of the radius of $(H)$ (Ths. $6,42,43$ ). From (xiv) we have the equation of $(H)$ as (xxxvi)

$$
n x^{2}-2 n H \cdot x+(n-1) H^{2}+1=0 .
$$

(ii) The polar prime of a point $P$ on $(O)$ w.r.t. $(H)$ divides the join of $H$ to the diametric opposite $P^{\prime}$ of $P$ in the ratio $1:(n-1)$. In fact, it is true for the quasi-polar hypersphere $(S)$ of a general $n$-simplex $\mathrm{S}(A)$ too w.r.t. its S-point $S$ (cf. Th. 11). Hence we have $(1 ; 11)$

(iii) The segment of an altitude of the OS between $H$ and the second point of intersection with $(O)$ is divided by its corresponding prime in the ratio $1:(n-1)$.

(iv) The $(n+1)$ paralles through $H$ to the joins of its vertices to $P$ meet its corresponding primes in $n+1$ points lying in the polar prime of $P^{\prime}$ w.r.t. $(H)$.

4.3. First $n(n+1)$ point-sphere. Now we have

THEOREM 51. An SoS in an n-space becomes an OS, if and only if the pair of its $N$-spheres coincide into the first $n(n+1)$ point-sphere $(N)$ of the OS passing through the $\left(\begin{array}{c}n+1 \\ 2\end{array}\right)$ midpoints of its edges and the $\left(\begin{array}{c}n+1 \\ 2\end{array}\right)$ feet of its bialtitudes there at with centre at its $F$-point $N=F_{n-3}$ (Ths. 41,49 (ii)).

4.4. $3(n+1)$ Point-sphere. From Theorem A follows

THEOREM 52. The $n+1$ Orthocentres and $n+1$ centroids of the prime faces of an $O S$ in an n-space lie on an hypersphere $\left(O^{\prime}\right)$ which cuts its altitudes again in the points dividing the segments between its Orthocentre and respective vertices in the ratio $1:(n-1)$.

4.5. Medial simplex. The medial simplex (14) formed of the centroids of the prime faces of an $O S$ in an $n$-space is homothetic w.r.t. their common centroid $G$, the homothetic ratio being $-1: n$, and is too therefore Orthogonal. If $H, H^{\prime}$ be the Orthocentres of the $O \mathrm{~S}$ and its medial simplex, we have (xxxvii)

$$
(n+1) G=n H^{\prime}+H .
$$

From (xii), (xxxiv), (xxxv), (xxxvii) follow the following

(xxxviii) $\quad n H^{\prime}=(n-2) H=n F_{1}, \quad$ or $\quad H^{\prime}=F_{1}$

(xxxix) $\quad 2 n O^{\prime}=n H^{\prime}+H+(n-1) H, \quad$ or $\quad 2 O^{\prime}=H+H^{\prime}$.

That is, $H^{\prime}$ coincides with the $F$-point $F_{1}$ of the $O S$ and is symmetric of $H$ w.r.t. the circumcentre $O^{\prime}$ of its medial simplex.

Further extending the idea of a pair of isogonal conjugate points $(11 ; 16)$ for a tetrahedron to those for an $n$-simplex, we can prove that (1):

"The $n+1$ pairs of projections, in the prime faces of a simplex, of a pair 
of its isogonal conjugate points lie on an hypersphere reith centre at the midpoint of their join."

Hence follows from the preceding Theorem the following

THeOREM 53. The medial simplex of an OS is Orthogonal such that its Orthocentre lies at the isogonal conjugate of that of the $O \mathrm{~S}$.

4.6. $2\left(\begin{array}{c}n+1 \\ p\end{array}\right)$ point-spheres. The GS-sphere $\left(O^{\prime \prime}\right)$ ( $(1.3 \mathrm{~b})$ of an $O S$ is referred to as its $G H$-sphere. From $\S \S 3.11,3.12$ now follow the following

THEOREM 54. The $\left(\begin{array}{c}n+1 \\ p\end{array}\right) G H$-spheres of the $(n-p)$-faces of an OS in an $n$-space lie on its $I$-sphere $\left(I_{p}\right)$ passing through their $\left(\begin{array}{c}n+1 \\ p\end{array}\right)$ Orthocentres and $\left(\begin{array}{c}n+1 \\ p\end{array}\right)$ centroids (Th. 44, $\left.I_{q}^{p}=I_{p}^{q}=I_{p+q}\right)$; The $\left(\begin{array}{c}n+1 \\ p-1\end{array}\right) 3(n-p+2)$-spheres of its $(n-p+1)$-faces lie on its $T$-sphere $\left(T_{p-1}\right)$ (Th. 46, $\left.T_{q}^{p}=T_{p}^{q}=T_{p+q}\right)$ coinciding with $\left(I_{p}\right)$ such that $\left(I_{1}\right)=\left(T_{0}\right)$ coincides with its $3(n+1)$ pointsphere $\left(O^{\prime}\right)$ (Th. 52), and $\left(I_{2}\right)=\left(T_{1}\right)$ with its SECOND $n(n+1)$ pointsphere, $(N)=\left(T_{n-2}\right)=\left(I_{n-1}\right)$ being the first (Th. 51); in fact, its $I$-spheres occur in pairs of homothetic $2\left(\begin{array}{c}n+1 \\ p\end{array}\right)$ point-spheres $\left(I_{p}\right),\left(I_{n-p+1}\right)(4<2 p<n+1)$ w.r.t. its centroid, homothetic ratio being $-p:(n-p+1)$.

THEOREM 55. An SoS in an n-space becomes an OS, if and only if the pair of its $L$ - or $k$-spheres coincide, i.e., the $\left(\begin{array}{c}n+1 \\ 3\end{array}\right)$ l-circles and $\left(\begin{array}{c}n+1 \\ 4\end{array}\right)$ second 12-point spheres of its tetrahedra lie on its L-sphere $(L)=\left(I_{n-2}\right)$, and the $\left(\begin{array}{c}n+1 \\ 5\end{array}\right)$ 15-point 3-spheres of its 4-simplexes lie on its $k$-sphere $(k)=\left(I_{n-3}\right)$ (Th. 45).

THEOREM 56 (i). The $\left(\begin{array}{c}n+1 \\ p\end{array}\right) G$-spheres of the $(n-p)$-faces of an OS in an $n$-space lie on its $F$-sphere $\left(F_{p}\right)$ with centre at its $F_{\text {-point }} F_{p}$ (Th. 49 (ii)) such that the $\left(\begin{array}{c}n+1 \\ 4\end{array}\right)$ first 12-point spheres of its tetrahedra lie on its first $n(n+1)$ point-sphere $(N)=\left(F_{n-3}\right)$ (Th. 51).

(ii) An SoS in an n-space becomes an OS, if and only it the pair of its $J$ spheres coincide into the $F$-sphere $\left(F_{n-2}\right)$ of the OS passing through the $\left(\begin{array}{c}n+1 \\ 3\end{array}\right)$ g-circles of its triangles (Th. 41).

THEOREM 57. The centre $I_{p}$ of the $I$-sphere $\left(I_{p}\right)$ of an $O S$ in an $n$-space divides the join of its Orthocentre $H$ to its circumcentre $O$ in the ratio $1:(n-p)$, and therefore the distances of $I_{p}\left(p=1, \cdots, n ; I_{n-1}=N, I_{n}=O\right)$ from $H$ form an harmonic progression. We may also note here that $I_{n-q}=F_{n-2 a-1}$.

For by Theorems 44, 49(ii) we have

$$
2 I_{p}=F_{p}+H=\frac{n-p-1}{n-p+1} H+H,
$$

or

(xl)

$$
(n-p+1) I_{p}=(n-p) H
$$

$O$ being the origin. 
4.7. Coaxal families. Every $p$-altitude of an $O S$ in an $n$-space meets its relative $(p-1)$ - and $(n-p)$-spaces in their Orthocentres (Th. 48) as a pair of inverse points for its polar hypersphere $(H)$ (Th. 50(i)). Thus from $\S 1.3 \mathrm{~b}$ and Theorem 47 now follow

TheOREm 58(i). All the I-spheres $\left(I_{p}\right)$ and the F-spheres $\left(F_{p}\right)$ of an OS in an $n$-space belong to the same coaxal family $f$, determined by its polar hypersphere $(H)$ and its circumhypersphere $(O)$, to which then belong its $G$-sphere $(G), 3(n+1)$ point-sphere $\left(O^{\prime}\right)$ and $G H$-sphere $\left(O^{\prime \prime}\right)$, and therefore $\left(I_{n-q}\right)=$ $\left(F_{n-2 q-1}\right)$ (Th. 57); in particular, its $N$-, $J$-, L- and $k$-spheres too belong to $f$ (cf. 11)

(ii) $(H)$ and $(G)$ form the pair of hyperspheres of antisimilitude for every pair of the $I$-spheres $\left(I_{p}\right)$ and $\left(I_{n-p+1}\right)$ of the $O S$, in particular of $(O)$ and $\left(O^{\prime}\right)$ for $p=n$ (Th. 54), and $\left(O^{\prime \prime}\right)$ is therefore their hypersphere of similitude and radical hypersphere of $(G)$ and $(H)$.

(iii) $(G)$ coincides with $\left(I_{p}\right)$ for $n=2 p-1$.

(iv) The radical prime of $f$ is the polar prime (16) of the Orthocentre $H$ of the $O S$ w.r.t. it or $(G)$ and therefore coincides with that of its centroid $G$ w.r.t. $(H)$. It follows by induction in the manner we have done for a 4-simplex (11).

(v) The vectorial equations of the various hyperspheres associated with the $O S$ can be now put down as follows: $(O),\left(O^{\prime}\right),(G),(H)$ as given by (ix), (x) (xv), (xxxvi);

$$
\begin{gathered}
(\mathrm{cf.}(\mathrm{xvi}))\left(O^{\prime \prime}\right) \equiv x^{2}-(G+H) \cdot x-G \cdot H=0 \\
\left(T_{p-1}\right)=\left(I_{p}\right) \equiv n(n-p+1) x^{2}-2 n(n-p) H \cdot x+(n-1)(n-p) H^{2}=p
\end{gathered}
$$

which reduces to $(O)$ for $p=n$, to $\left(O^{\prime}\right)$ for $p=1$ and to $(G)$ for $n=2 p-1$

$$
\begin{gathered}
\left(F_{p}\right) \equiv n(n-p+1) x^{2}-2 n(n-p-1) H \cdot x+(n-1)(n-p-1) \\
H^{2}=n+p+1 \\
(N)=\left(I_{n-1}\right)=\left(F_{n-3}\right) \equiv 2 n x^{2}-2 n H \cdot x+(n-1) H^{2}=n-1 \\
(J)=\left(F_{n-2}\right) \equiv 3 n x^{2}-2 n H \cdot x+(n-1) H^{2}=2 n-1 \\
\left(F_{n-5}\right)=(L)=\left(I_{n-2}\right) \equiv 3 n x^{2}-4 n H \cdot x+2(n-1) H^{2}=n-2 \\
\left(F_{n-7}\right)=(k)=\left(I_{n-3}\right) \equiv 4 n x^{2}-6 n H \cdot x+3(n-1) H^{2}=n-3
\end{gathered}
$$

4.8. Orthocentric set. If $A_{n+1}=H$ be the Orthocentre of an $O S=A_{0} \cdots A_{n}$, the $n+2$ points $A_{i}(i=0, \cdots, n+1)$ are said to form an Orthocentric Set such that every simplex formed of any $n+1$ of them is obviously Orthogonal with the $(n+2)$ nd point as its Orthocentre. As an immediate consequence of the definitions now follows the following

Theorem 59. The $n+2$ Euler lines of the simplexes formed of an Orthocentric Set of $n+2$ points CONCUR at their CENTROID E, referred to 
as their ORTHIC POINT (cf. 1) such that their $n+2$ centroids or circumcentres too form an Orthocentric set homothetic to the given one w.r.t. $E$, the homothetic ratios being $-1:(n+1)$ and $-n: 2$.

For

$$
(n+2) E=(n+1) G_{i}+A_{i}=n A_{i}+2 O_{i} \quad(\text { Th. } 49(\mathrm{i})),
$$

$O_{i}, G_{i}, A_{i}$ being the circumcentre, centroid and Orthocentre of the simplex formed of the $n+1$ points of the given Group other tian $A_{i}$.

THEOREM 60. If $n+2$ hyperspheres are mutually orthog al, their centres form an Orthocentric set such that each is the polar hyperspl of the simplex formed of the centres of the rest.

For the centres of any two form a pair of conjugate point; for every other.

Thanks are due to Prof. T. G. Room for his kind directusns and to M/S R. P. Goel, R. K. Datta and N. P. Jaggi (Students at the Indian Institute of Technology, Kharagpur) for tracing the figures.

\section{References}

[1] Court, N. A., Modern pure solid geometry (New York, 1935).

[2] Court, N. A., On the theory of the tetrahedron, Bul. Amer. Math. Soc. 48 (1942), 583-589

[3] Court, N. A., The semi-orthocentric tetrahedron, Amer. Math. Mon. 60 (1953), 306 - 310.

[4] Coxeter, H. S. M., Editorial note to solution of problem 3963, ibid 49 (1942), 133.

[5] Coxeter, H. S. M., Editorial note to solution of problem 4049, ibid. 50 (1943), 567-578.

[6] Coxeter, H. S. M., Regular polytopes (London, 1948).

[7] Mandan, S. R., Umbilical projection in four dimensional space $\mathrm{S}_{4}$, Proc. Ind. Acad. Sc. A 28 (1948), $166-172$.

[8] Mandan, S. R., Spheres associated with a semi-orthocentric tetrahedron, Res. Bul. Panj. Uni. 127 (1957), 447-451.

[9] Mandan, S. R., An S-configuration in Euclidean and elliptic $n$-space, Can. J. Math. 10 (1958), $489-501$.

[10] Mandan, S. R., Altitudes of a simplex in four dimensional space, Bul. Cal. Math. Soc. 50 (1958 Supp.), 8-20.

[11] Mandan, S. R., Semi-orthocentric and Orthogonal simplexes in a 4-space, ibid., $21-29$.

[12] Mandan, S. R., Altitudes of a general 4-simplex, ibid., 34-4l.

[13] Mandan, S. R., Uni- and Demi-orthocentric simplexes, Jour. Ind. Math. Soc. 23 (1959), 169-184.

[14] Mandan, S. R., Medial simplex, Math. St. 28 (1960), 49-52.

[15] Mandan, S. R., Uni- and Demi-orthocentric simplexes II, Journ. Ind. Math. Soc. 25 (1961).

[16] Mandan, S. R., Altitudes of a general $n$-simplex, Jour. Australian Math. Soc. (in press).

[17] Mandan, S. R., Polarity for a simplex, ibid. 